\title{
High frame rate contrast enhanced ultrasound imaging for slow flow: influence of ultrasound pressure and flow rate on bubble disruption and image persistence
}

Jiaqi Zhu ${ }^{1}$, MEng • Shengtao Lin ${ }^{1}, \mathrm{PhD} \cdot$ Chee Hau Leow ${ }^{1}, \mathrm{PhD} \cdot$ Ethan M. Rowland ${ }^{1}, \mathrm{PhD}$ • Kai Riemer ${ }^{1}$, MSc • Sevan Harput ${ }^{1}, \mathrm{PhD} \cdot$ Peter D Weinberg ${ }^{1}, \mathrm{PhD} \bullet$ Meng-Xing Tang ${ }^{1, *}$, $\mathrm{PhD}$

\footnotetext{
${ }^{1}$ Department of Bioengineering Imperial College London

Exhibition Road

London SW7 2AZ

United Kingdom

*Corresponding author Dr. Meng-Xing Tang

Department of Bioengineering, Imperial College London, Exhibition Road, London SW7 2AZ, United Kingdom Tel: + 44 (0)2075943664 mengxing.tang@imperial.ac.uk
} 


\section{Abstract}

Contrast enhanced ultrasound (CEUS) utilising microbubbles shows great potential for visualising lymphatic vessels and identifying sentinel lymph nodes (SLN) which is valuable for axillary staging in breast cancer patients. However, current CEUS imaging techniques have limitations that affect the accurate visualisation and tracking of lymphatic vessels and SLN. (1) Tissue artefacts and bubble disruption can reduce the image contrast. (2) Limited spatial and temporal resolution diminishes the amount of information that can be captured by CEUS. (3) The slow lymph flow makes Doppler based approaches less effective. This work evaluates on a lymphatic vessel phantom the use of high frame-rate (HFR) CEUS for the detection of lymphatic vessels where flow is slow. Specifically the work particularly investigates the impact of key factors in lymphatic imaging, including ultrasound pressure and flow velocity as well as probe motion during vessel tracking, on bubble disruption and image contrast. A trail was also conducted to apply HFR CEUS imaging on vasculature in a rabbit popliteal lymph node (LN). Our results show that (1) HFR imaging and SVD filtering can significantly reduce tissue artefacts in the phantom; (2) the slow flow rate within the phantom makes image contrast and signal persistence more susceptible to changes in ultrasound amplitude/MI, and an MI value can be chosen to reach a compromise between images contrast and bubble disruption under slow flow condition; (3) probe motion significantly decreases image contrast of the vessel, which can be improved by applying motion correction prior to SVD filtering; (4) the optical observation of the impact of ultrasound pressure in HFR CEUS further confirm the importance of optimising ultrasound amplitude MI; (5) Vessels inside rabbit LN with blood flow less than $3 \mathrm{~mm} / \mathrm{s}$ are clearly visualised.

\section{Keywords}



decomposition; Motion correction

\section{Introduction}

Lymphatic system plays a crucial role in cancer metastasis. In breast cancer, the axillary lymphatic system acts as an avenue in which cancer cells spread. Cancer cells invade small

7 lymphatic vessels near the primary tumour and migrate with the lymphatic fluid. Lymph nodes linking the lymphatic vessels play a critical role in filtering the cancer cells. Thus, the presence of metastases in axillary lymph nodes is an important indicator for breast cancer staging. Among these axillary lymph nodes, the SLN is the first lymph node that receives the lymphatic fluid from breast tumour. If the SLN is free of metastases, there is a high possibility that the cancer cells have not spread to the other lymph nodes (Somasundaram et al. 2007). Therefore, nodal status of axillary SLNs is essential for determining the adjuvant treatment strategy for patients diagnosed with breast cancer (Pesek et al. 2012). Currently a surgical procedure called sentinel lymph node biopsy is routinely used to track lymphatic vessels and identify and dissect the SLN (Pesek et al. 2012; Veronesi et al. 2003). However, the invasive test procedure causes a 4\% $14 \%$ rate of complications (Temple et al. 2002) and shows variable accuracy affected by time constraint (Fujishima et al. 2009; Wong et al. 2015). Hence, there is a strong clinical need for an effective pre-operative and non-invasive technique to identify SLN in breast cancer patients. identification of lymphatic vessels and SLN in breast cancer patients (Cox et al. 2013; Goldberg et al. 2004; Sever et al. 2011). CEUS is an imaging technique which utilises microbubbles as contrast agents to allow better visualisation of the vasculature and flow. When microbubbles are exposed to ultrasound field, they produce backscattered signals containing fundamental and 
1 harmonic components, which greatly increase the ultrasound echoes from within the vessels.

2 Microbubbles have been widely used in clinical diagnosis and are safe with little reported side effect or complications. CEUS allows the preoperative identification of SLN and when combined with image guided needle biopsy, serves as a promising axillary nodal staging method.

There are several studies on the use of CEUS for the lymphatic system. The technique was first described by Goldberg et al. in several swine melanoma model (Goldberg et al. 2004). Sever et al. (Sever et al. 2011) utilised CEUS for the visualisation of lymphatic vessels and SLNs in patients with diagnostic breast cancer. After the periareolar intradermal injection of microbubbles, they successfully monitored the complete process of lymph draining which starts at the afferent lymphatic vessel and flows through the lymph node and finally exits through the efferent vessel. In their most recent study (Cox et al. 2013), CEUS was used to guide percutaneously SLN biopsy after preoperative detection of SLN in 295 breast cancer patients. With the help of CEUS imaging, a secondary surgical procedure was avoided in 29 patients.

Although the feasibility of SLN identification by tracking microbubbles transporting through lymphatic vessels was confirmed by these encouraging results, tissue artefacts and bubble disruption can reduce the contrast of lymphatic vessels, compromising the image quality. The failure rate of SLN detection ranges between $4 \%$ (Cox et al. 2013) and 11\% (Sever et al. 2011) even with experienced operators. Different CEUS imaging techniques such as pulse inversion (PI) (Simpson et al. 1999) and amplitude modulation (Brock-Fisher et al. 1996) are developed to distinguish microbubble and tissue signals by detecting nonlinear echoes arising from microbubble oscillations. However, nonlinear echoes can also be produced by nonlinear propagation of ultrasound in tissue or bubble cloud (Tang et al. 2010). This can misrepresent tissue as microbubbles in the final image, forming nonlinear tissue artefacts. These artefacts reduce image contrast, particularly at high clinical ultrasound frequencies where the sensitivity 
1 of microbubble detection is lower than that of low clinical frequencies (Tang et al. 2010), and

2 lead to inaccuracies in bubble tracking and SLN identification. Significant tissue nonlinear artefacts are observed in lymph vessels imaging (Fig. 1) using commercial system. Furthermore, the lymphatic flow is much slower than arterial flow, making it more difficult to detect and separate from tissue. Finally the imaging probe has to be manually moved to follow the lymphatic vessel track in order to identify the sentinel lymph node(s) and the additional motion between the probe and tissue may further impact the image quality. These factors can increase variability, leading to greater operator dependency.

Recent advances in HFR ultrasound imaging (Tanter and Fink 2014), together with spatiotemporal clutter filtering, can potentially help to address the above. Compared to conventional line-by-line focus imaging, HFR ultrasound imaging (Montaldo et al. 2009) with frame rate typically two orders of magnitude higher than conventional ultrasound can be achieved based on transmitting unfocused waves and parallel beamforming. Furthermore, coherent summation of varied angle plane wave transmission is able to improve the image quality and make up for the lack of focus transmission (Denarie et al. 2013). Enhanced contrast is achieved by plane wave images at the same disruption ratio of focus images, when detecting microbubbles (Couture et al. 2012). Thanks to the dramatic increase in the number of temporal samples in ultrafast plane wave imaging, advanced spatial temporal analysis such as singular value decomposition (SVD) filtering can be used to remove dominant artefact in blood/microbubble flow imaging arising from slow moving but relatively static tissue. In contrast to traditional high pass filtering, SVD filter investigates both temporal and spatial characteristics of tissue and blood/microbubble signals. While the effectiveness of SVD filter for detection of small vessels with tissue motion has been demonstrated in ultrafast Doppler blood imaging and HFR CEUS imaging (Demené et al. 2015b; Demené et al. 2016; Desailly et al. 2017; van der Ven et al. 2017). The complex relationship among MI, flow velocity, bubble 
1 disruption and image contrast, particularly in the context of lymphatic flow, have not been

2 investigated.

In this study, we apply SVD filter on HFR CEUS for the visualisation of slow flow on a lymphatic mimicking phantom. In-vitro experiments on lymphatic mimicking phantom are designed to understand the impacts of lymph flow velocities, ultrasound pressures and probe motion on HFR CEUS image contrast. Our study indicates the trade-off between minimizing microbubble disruption and achieving required contrast for detecting microbubble from tissue signals, and shows the need for optimising ultrasound pressure for different flow velocities. HFR and optical microscope were used to observe the impact of the MI of HFR ultrasound on bubble disruption. Preliminary studies were conducted on imaging a rabbit lymph node microvasculature.

\section{Materials and Methods}

\section{$\underline{\text { In vitro experiment set-ups and ultrasound parameters }}$}

A lymph vessel mimicking phantom (Fig. 2a) was made consisting of a $300 \mu \mathrm{m}$ tube (Platinum Cured Silicone Tube, Harvard Apparatus UK, Cambridge, United Kingdom) inside a tissue-mimicking phantom (Madsen et al. 1998), which was made of agar containing glass beads (45-90 $\mu \mathrm{m}$ in diameter, Potters Ballotini Ltd, Barnsley, South Yorkshire, United Kingdom) as scatterers. The diameter of the tube was chosen according to the average size of the afferent lymphatic vessels in human (Pan et al. 2010). The average depth of tube was set to $1.6 \mathrm{~cm}$ from the top surface, which mimicked the average depth between the axillary lymphatic vessel and the skin (Bentel et al. 2000). Flowing microbubbles of concentration $\sim 2.5 \mathrm{x} 10^{7} \mathrm{MB} / \mathrm{mL}$ were administered using a syringe pump. The microbubbles used for all the experiments were inhouse lipid-shell microbubbles filled with decafluorobutane which were manufactured according to the formula described in (Lin et al. 2017). 
2 Vantage 128, Verasonics, Kirkland, USA), a linear probe L12-3v (192-element linear array transducer, Verasonics, Kirkland, USA) and a 5-angle compounding plane wave imaging sequences. The transmitted pulses were centred at $4 \mathrm{MHz}$ with a pulse repetition frequency of $5000 \mathrm{~Hz}$ for all the ultrasound acquisitions. The acquired radio frequency (RF) data were beamformed to yield 1000 frames of compounded B-mode images per second which were then processed by SVD filter. All in vitro experiments in this paper are repeated three times unless otherwise stated.

After SVD, microbubble and tissue signals can be represented by different sets of singular vectors with independent spatiotemporal coherence. Among these vectors, large singular values correspond to tissue signals with high temporal and spatial coherence, while lower components are associated with blood signals with relatively low spatiotemporal coherence. Based on this assumption, distinction between clutter and blood/microbubble signals can be achieved by setting threshold on the singular values. For all the B-mode images in this study, components corresponding to higher singular values, which represent tissue signals, were filtered out. The SVD cut-off thresholds were chosen so as to achieve the maximum contrastto-tissue ration (CTR) (Song et al. 2017).

In order to compare the performance of SVD in combination with HFR imaging and standard CEUS technique - PI in suppressing tissue artefacts, a two-tube set-up (Fig. 2b) was made consisting of a $200 \mu \mathrm{m}$ cellulose tube (Cuprophan, RC55 8/200 Membrana GmbH, Germany) filled with non-flowing microbubbles, and another tube filled with water as control. Plane wave B-mode images were obtained with the same ultrasound settings and were subsequently processed by SVD filter. Plane wave PI images were acquired with the same compounding angles and transmit frequency as B-mode images. 


\section{In vitro ultrasound experiment: effect of flow rate and ultrasound pressure}

The effect of different flow velocities in lymphatic vessels and different ultrasound pressures was investigated. To create a range of velocities similar to lymphatic flow, a programmable syringe pump (Harvard Apparatus Programmable PHD2000 pump, Harvard Apparatus UK, Cambridge, United Kingdom) was connected to one side of the tube to control the mean flow velocities of microbubbles at $4,10,20,40$ and $80 \mathrm{~mm} / \mathrm{s}$. These flow velocities were chosen according to the different average lymph flow rate found in the main lymphatic trunks of human (1999). These flow rates ranged from the slower $0.7 \mathrm{~mm}^{3} / \mathrm{sec}$ (flow velocity 4 $\mathrm{mm} / \mathrm{s}$ ) of the subclavian lymphatic vessels to the faster $14 \mathrm{~mm}^{3} / \mathrm{sec}$ (flow velocity $80 \mathrm{~mm} / \mathrm{s}$ ) of the intestinal vessels. Furthermore, three different ultrasound pressures (mechanical index (MI)) were applied $(\mathrm{MI}=0.050 .11,0.22)$. The lowest $\mathrm{MI}$ value $(0.05)$ minimises microbubble destruction. Moderate MI value (0.11) and relatively high MI value (0.22) may introduce different levels of microbubble disruption. The varying microbubble disruption events and contrast signal persistence under different ultrasound amplitudes were further studied in an optical experiment.

\section{$\underline{\text { In vitro ultrasound experiments: probe motion }}$}

This experiment studied the effect of probe motion on HFR CEUS imaging of the lymphatic vessel phantom. To simulate relevant probe motion (Desailly et al. 2017), the ultrasound probe was mounted on a linear motor and the movement of the probe was set at 2 $\mathrm{mm} / \mathrm{s}$ in the longitudinal direction along the tube. Two opposite directions (along/against the microbubble flow direction) of movement were randomly repeated during acquisition. The total displacement of the probe was set to $6 \mathrm{~mm}$ to ensure the travelling time was longer than the ultrasound data acquisition time. All the flow velocities used in the previous flow velocity experiment were tested under probe motion. Moderate MI value $(0.11)$ was used in this experiment since it provides the highest contrast and persistence. 
For the images acquired with probe motion, two different processing methodologies were applied and investigated. One of the methods utilised SVD filter which was the same as that used for images from the static probe. The other method corrected for the motion induced by the moving probe before application of SVD filter. The motion correction was performed based on a rigid image registration algorithm (Harput et al. 2017). The motion estimation was performed on the original B-mode image after normalisation and log-compression of the complex magnitude of beamformed data. Then the transformation matrix was applied to the real and imaginary part of the corresponding beamformed data separately. SVD filter was subsequently used to process the motion-corrected complex data.

\section{Optical experiment}

Optical experiment was conducted to observe the microbubble behaviours under different ultrasound pressures during HFR CEUS imaging. Observation was done by using an optical microscope and recorded with a high speed camera using the set-up shown in Fig. 3. The water immersion objective lens (100×, NA=1.0, LUMPlanFL, Olympus, Tokyo, Japan) was connected to a high-speed camera (FASTCAM SA5, Photron, San Diego, USA) synchronised with Verasonics system, in which optical images were recorded at 12000 frames per second. Optical path and ultrasound field were set up normal to each other to avoid the obstruction arising from objective in propagation of ultrasound wave. Microbubble solution of the same concentration $\left(\sim 2.5 \times 10^{7} \mathrm{MB} / \mathrm{mL}\right)$ were introduced into a $200 \mu \mathrm{m}$ acoustically and optically transparent cellulose-tube. That tube was placed at the focal depth of the microscope, where the corresponding MI was calibrated. Both ends of the tube were clipped to stop any backflow. Clips were removed to allow freely flowing microbubbles to replenish when a new experiment starts. Acquisition of ultrasound and optical data started simultaneously after 20 seconds of microbubble injection. Each frame of the original image from the high speed camera has a size of $128 \times 128 \mu \mathrm{m}^{2}$. In order to focus on the regions containing microbubbles, the images 
1 presented in the result section were cropped from the original images to a size of $47.24 \times 47.24$

$2 \mu \mathrm{m}^{2}$ for better visualisation of the whole process.

$3 \quad$ In vivo ultrasound experiments

All animal experiments complied with the Animals (Scientific Procedures) Act 1986, were approved by the Local Ethical Review Panel of Imperial College London, and carried out under Home Office License P15180DF2L. A healthy male New Zealand rabbit (HSDIF strain, Envigo, Hillcrest, U.K.) aged 6-8 weeks was used in this study. Hypnorm $(0.3 \mathrm{~mL} / \mathrm{kg})$ and midazolam $(0.025 \mathrm{~mL} / \mathrm{kg})$ were administered via the marginal vein to produce general anaesthesia. Fur on the legs of the rabbits was removed and rabbits were placed on a warming plate to maintain the body temperature at $37^{\circ} \mathrm{C} .0 .1 \mathrm{~mL}$ Evans blue dye was injected subcutaneously into the rear footpad to label the lymphatic drainage of the hind leg. Gentle massage was administrated on leg and rear footpad to accelerate the flow of the dye into lymphatic channels which drained into the popliteal LN. After 5 to 10 mins of Evans blue dye administration, the blue-labelled lymphatic channels and popliteal LN were identified after cutting/opening the first layer of the skin at the back of the knee. $0.5 \mathrm{~mL}$ lignocaine was injected previously and locally into the skin at the back of the knee to apply local anaesthetic on the right leg. A bolus injection of $0.1 \mathrm{ml}$ in-house microbubbles was introduced through the marginal ear vein. The in-house microbubbles used in rabbit experiments were the same as those used in in-vitro experiment with same concentration.

HFR CEUS imaging sequence was implemented into a research platform (Verasonics Vantage 128, Verasonics, Kirkland, USA) equipped with a linear probe L22-14v (128-element linear array transducer, Verasonics, Kirkland, USA). Plane waves with 15 inclination angles between $-7.5^{\circ}$ and $7.5^{\circ}\left(1^{\circ}\right.$ angle step) were transmitted at a pulse repetition frequency of 25 $\mathrm{kHz}$ to obtain coherently compounded B-mode images at a frame rate of $800 \mathrm{~Hz}$. For each angle, a single-cycle plane wave pulse was transmitted at $18 \mathrm{MHz}$ and a mechanical index of 
1 0.11. The acquired radio frequency (RF) data were beamformed to yield 800 frames of

2 compounded B-mode images which were then processed by SVD filter. The filtered data were

3 temporally correlated to generate high contrast vascular images. Furthermore, estimation of

4 frequency shift generated the direction and velocity of blood flow inside the imaging node

5 which were shown on directional image.

6

7

8

9

10

\section{Quantitative evaluation}

We used two measures to evaluate the image quality under the effect of flow velocity, probe motion and ultrasound pressure. The two measures are CTR and integral of normalised intensity (INI).

CTR of one single frame was computed as the mean pixel intensity of the region of microbubbles over that of the surrounding tissue. The CTR was averaged over $1 \mathrm{~s}$ to obtain the mean CTR. Integral of INI was proposed to measure the persistence of contrast signals over time. INI of a video sequence was calculated as follows: 1) Mean intensity of the vessel region was obtained for each frame over the acquisition time of 1s. 2) Mean intensity from step one was normalised by the mean intensity of the first frame of the video so that the intensities at $t=0$ is 1 for all video sequences. 3) Normalised intensities from step 2 were integrated over the entire time interval of $1 \mathrm{~s}$ to obtain INI. The computation was given by

$$
I N I=\sum_{1}^{N} \frac{\text { mean intensities of bubble at frame no. } i}{\text { mean intensities of bubble at frame no. } 1}
$$

where $\mathrm{N}$ is the total frame number. The ability for retaining contrast signals over time regardless of initial amplitude can be captured by INI due to the normalisation process.

The region of interest (ROI) for microbubbles was chosen according to the shape of the tube in the lymphatic vessel phantom. The ROI for background tissue was set to be $3 \mathrm{~mm}$ above that of ROI for microbubbles. In the experiment for investigating the effect of flow velocities 
1 and ultrasound pressure, both ROIs were fixed throughout the sequence as there was no motion

2 among all the acquired frames. In the experiment for the effect of probe motion, the same fixed

3 ROIs were used in the case of images after motion correction associated with SVD filter. But

4 CTRs of images processed by SVD filter without motion correction was calculated based on

5 moving ROIs. For each video acquired with the moving probe, automatic motion tracking of the

6 ROIs was performed using rigid image registration. A transformation matrix was derived by

7 rigidly registering each image in the sequence to the first image. The manual ROIs on the first

8 image can then be propagated to the other images in the sequence using these transformation

9 matrices to obtain the motion-corrected ROIs.

In the optical observation, ratio of the disrupted microbubble to the total number of microbubbles was used to investigate the behaviour of microbubbles. The total number of microbubbles was measured on the original optical images before insonifying plane wave ultrasound. The number of disrupted microbubbles was recorded from the start of acquisition to $50 \mathrm{~ms}$ and $550 \mathrm{~ms}$. The disruption of microbubbles is defined as changes of microbubbles including coalescence, fragmentation, shrink and destruction. The disruption ratio for each of the three MIs was calculated by firstly calculating the ratio for each video data set and then averaged across three video data sets.

\section{Results}

\section{$\underline{\text { In vitro ultrasound experiment: comparison between PI and SVD filter }}$}

SVD filter and PI techniques were qualitatively compared in terms of tissue artefacts. In

Fig.4, the SVD filtered image was able to detect bubbles from the bottom tube while removing signals from the top control tube that was present in the PI image. In order to make a fair comparison, all the ultrasound images presented in this paper have a 40dB dynamic range. 
1 In vitro ultrasound experiment: effect of flow rate and ultrasound pressure

Qualitative evaluation was performed by visual observation of the images obtained from the STA of the beamformed images. Fig. 5 a-e showed the images for different flow rates (4, $10,20,40,80 \mathrm{~mm} / \mathrm{s}$ ) at a fixed MI of 0.05 . Improved contrast signals can be observed at higher flow velocities. Fig. 6 and Fig. 7 compared images obtained using three different MIs (0.05, 0.11 and 0.22 ) at fixed flow velocities of $4 \mathrm{~mm} / \mathrm{s}$ and $10 \mathrm{~mm} / \mathrm{s}$.

Quantitative evaluation was conducted using CTR and INI. Fig. 8a illustrated the effects of different flow velocities and MIs on the mean CTR. We observed that an increase in the flow velocities results in a corresponding increase in mean CTR for all MI values, which was consistent with the qualitative results reported in Fig. 5. Fig. 8a also indicated that higher MI increases mean CTR at all velocities except for the lowest flow rate $(4 \mathrm{~mm} / \mathrm{s})$, in which a moderate MI achieves the highest value. Fig. $8 \mathrm{~b}$ revealed how INI changes with MI depicting a decrease of INI with increasing MI. INI values were averaged over 5 flow velocities.

\section{$\underline{\text { In vitro ultrasound experiment: probe motion }}$}

Qualitative evaluation under probe motion was conducted on the images after treatment of SVD filtering. Fig. 9a-f presented the images without probe motion and with probe speed of $2 \mathrm{~mm} / \mathrm{s}$ at a fixed MI of 0.11 and under varied flow rates. The image contrast declined due to the moving probe at different flow velocities. CTR was first acquired from the SVD filtered images to quantitatively assess the effect of probe motion. Fig. 10a compared the CTRs acquired with the static probe and the moving probe. The significant decrease in CTR in the presence of motion can be clearly observed at all flow velocities. Both static and moving probe gave similar results that CTR increases with increase in flow velocity. 

reported the CTR before and after the application of motion correction algorithm. For the lower flow velocities of $4 \mathrm{~mm} / \mathrm{s}$ and $10 \mathrm{~mm} / \mathrm{s}$, motion correction results in higher CTR.

4

5

\section{$\underline{\text { Optical experiments }}$}

In this section, we investigated the behaviour of microbubbles when insonified with ultrafast compounding plane waves at different MIs, to better understand the contrast signal intensity persistence observed experimentally. Different phenomena were observed in microscopic images at different ultrasound pressures, capturing various microbubble behaviour such as coalescence, disappearance, repeated fragmentation and coalescence, bouncing and translation. The first frames in all of the presented optical figures were acquired at 0ms before the arrival of ultrasound wave at the focus zone in the tube.

Fig. 11a and Fig. 11b displayed the rapid changes of microbubbles at the higher MI of 0.36. In Fig. 11a, multiple coalescence, shrinking and translation of microbubbles were recorded. During the first $3.58 \mathrm{~ms}$, microbubble no. 1 , no. 3 and no. 4 moved towards each other and subsequently coalesced under the secondary radiation force. Microbubble no.6 and no.8 shrank and finally disappeared at $5.08 \mathrm{~ms}$. Microbubble movement in the direction of ultrasound field under the primary radiation force was clearly observed by the upward displacement of the agglomerate of multiple microbubbles from 5.08 to $18.17 \mathrm{~ms}$. The horizontal dashed lines at 5.08 and $6.08 \mathrm{~ms}$ represented the same depth in the full original images acquired by the camera. In Fig. 11b, repetitive fragmentation and coalescence, shrinking and translation of microbubbles were shown. The original microbubble denoted with white arrow at $0 \mathrm{~ms}$ broke up into two microbubbles after $2.33 \mathrm{~ms}$ of ultrasound insonification. The two fragmented microbubbles coalesced at $2.5 \mathrm{~ms}$ and re-split at $2.67 \mathrm{~ms}$. These alternately repeated fragmentation and coalescence process appeared in 8 sequences until $4.92 \mathrm{~ms}$. 
At the MI of 0.22 , the main observed behaviour of microbubbles was coalescence. In

2 Fig. 11c, two pairs of microbubbles coalesced with different speed due to the different initial

distances between microbubbles. The initial distance (at $0 \mathrm{~ms}$ ) between the two microbubbles in the top row was larger than that in the bottom row. Hence, it took the microbubbles $28.58 \mathrm{~ms}$ to change in the top row, while only $8.25 \mathrm{~ms}$ in the bottom row. Both processes of microbubble coalescence were recorded which begin with bubble collision $(24.5 \mathrm{~ms}$ in the top row and 2.08 $\mathrm{ms}$ in the bottom row), followed by flattening of contact surfaces and film rupture, the formation of ellipsoidal bubbles ( $24.83 \mathrm{~ms}$ in the top row and $5.58 \mathrm{~ms}$ in the bottom row), and finally to the spherical bubbles (28.58 $\mathrm{ms}$ in the top row and $8.25 \mathrm{~ms}$ in the bottom row) (Postema et al. 2004a).

At the MI of 0.11 , most of the microbubbles clustered and did not further coalesce until the end. Fig. 11d showed the example of clustering of multiple microbubbles. At this MI of 0.11, although most microbubbles did not further combine until the end, there was still an example of coalescence at slower speed observed in Fig. 11e. It took $47.08 \mathrm{~ms}$ to become the final spherical bubble at $\mathrm{MI}=0.11$ while less than $4 \mathrm{~ms}$ for $\mathrm{MI}=0.36$ and between $8.25 \mathrm{~ms}$ and $28.58 \mathrm{~ms}$ for $\mathrm{MI}=0.36$.

For the MI values below and equal to 0.05 , microbubbles remained stable and none of the above behaviours were recorded during the experiments.

Table 1 provides a direct comparison of microbubble behaviour at different ultrasound pressures. The study shows that ultrasound pressure significantly influences microbubble disruption, causing $100 \%$ disruption when $\mathrm{MI}=0.36$, and $23 \%$ of disruption when $\mathrm{MI}$ is as low as 0.1 . 
Qualitative evaluation was performed by applying temporal average of consecutive

3

original B-mode images and processed images. Fig. 12a reveals the grey-scale ultrasound image. The white dashed circle indicated the popliteal lymph node. From the same RF data, the vascular image (Fig. 12b) generated by SVD enabled clearer visualisation of the rich intra-nodal vasculature. Directional image where colours indicate the direction of flow are generated by detecting frequency shift. As illustrated by Fig. 12c, blue colour indicates flow going towards the transducer and red colour indicates flow going away from the transducer. The colourmap reveals that the majority of blood flow velocities range from 0 to $3 \mathrm{~mm} / \mathrm{s}$. White arrows in all the subfigures indicate the major vessels. Those major vessels can only be detected in the vascular and directional images (Fig. 12b and c) while is not visible in the B-mode image. In the directional image, differentiation between flow direction and speed can separate the opposing blood flow into arteries and veins, and therefore enhance the visualisation of vessels within the vascular image which are not spatially separable.

\section{Discussion}

In this study, HFR ultrasound imaging coupled with advanced processing techniques have been investigated for lymphatic system imaging on a lymphatic vessel phantom. Three influencing factors (flow velocity, probe motion and ultrasound pressure/MI) were studied. The results show that while HFR ultrasound and spatial temporal analysis can significantly reduce tissue artefacts, image contrast can still be low when imaging slow lymphatic flow under probe motion. The results show that ultrasound pressure plays a critical role in achieving high contrast and better persistent signal in tracking the slow-flowing lymph to locate SLN. Furthermore, motion correction is shown to be able to reduce the artefacts induced by probe motion. Results 
1 from these experiments will help us in the understanding and development of an accurate,

2 sensitive and operator-independent imaging technique capable of detecting lymphatic vessels

3 and SLN. Preliminary results from rabbit popliteal LN demonstrated the efficiency of HFR

$4 \quad$ CEUS and SVD filter in detecting vasculature with slow blood flow.

$5 \quad$ In vitro ultrasound experiment: comparison between PI and SVD filter

6

Nonlinear tissue artefacts are common in commercial ultrasound system at high clinical ultrasound frequency. Even at low MI, the nonlinear wave propagation may contribute to the artefact as demonstrated by Tang et al. (Tang et al. 2010) using a commercial systemIn the two tube set-up, the harmonic signals from the tube itself are strong. Due to the reduction of harmonic signals from microbubbles when the driving frequency (4 $\mathrm{MHz}$ in this study) is beyond bubble resonance frequency, the contrast signals are weak and results in the tissue artefacts on the PI image. System nonlinearity may also contribute to the tissue artefact. On the other hand, SVD filter dose not rely on the nonlinear components to extract microbubbles from tissue. Hence, SVD filtered image was able to detect bubbles from the bottom tube while removing static signals from the top control tube in this case.

\section{$\underline{\text { In vitro uUUltrasound experiment: effect of flow rate and pressure }}$}

For the effect of flow velocity, both qualitative (Fig. 5) and quantitative results (Fig. 8a) demonstrate the gain in contrast resulting from increased flow rate. This is because the distinction between microbubble and tissue with SVD filter relies on the different spatiotemporal coherence levels. The temporal characteristics and spatial distribution of fast flowing microbubbles and static tissue speckles differ widely, resulting in enhanced CTR. But for the slow flowing microbubbles, the difference from static tissue is insignificant, resulting in low CTR. 

disruption and required contrast for detecting microbubble from tissue signals. Disruption of microbubbles by different pressures of plane wave imaging has been acoustically studied by

4 Olivier et al (Couture et al. 2012). Compared to conventional focus imaging, plane-wave 5 imaging achieved better contrast at a specific disruption ratio. However, the relative effects of 6 flow rate and ultrasound pressure have not been evaluated. It appears that the effect of ultrasound 7 pressure differs under various flow velocities. and $10 \mathrm{~mm} / \mathrm{s}$. However, towards the later stage of the acquisition at $550 \mathrm{~ms}$, we observed better contrast using moderate MI $(0.11)$ at $4 \mathrm{~mm} / \mathrm{s}$. At $10 \mathrm{~mm} / \mathrm{s}$, higher MI still gives better contrast. This explains the different results of CTR according to MI under distinct flow velocities. In Fig. $8 \mathrm{a}$, at $4 \mathrm{~mm} / \mathrm{s}$, the highest value of CTR is given by the moderate MI and the lowest value is acquired by the lowest MI. In other cases of higher flow, higher CTRs are achieved by higher MI. This is because increase in ultrasound pressure introduces varied microbubble disruption behaviour, especially with large amount of microbubbles at the beginning of the acquisition. These microbubble changes are able to produce dissimilarity in temporal characteristics and spatial distribution (i.e. spatiotemporal coherence) between microbubble and tissue signals, resulting in improved efficiency of STA. A higher MI gives much higher contrast signals at the beginning of the acquisition due to the violent and rapid changes of microbubbles. It also tends to destroy the bubbles faster, causing contrast signals to decrease sharply and they cannot be retained over a long time as shown in Fig. 6f. The low persistence of signals achieved by higher MI can be further confirmed by INI. In Fig. 8b, larger INI with lower MI indicates that higher ultrasound pressure leads to shorter retaining time of contrast signal. This is consistent with a smaller mean CTR value obtained at $4 \mathrm{~mm} / \mathrm{s}$ which accurately captures such decay. However, faster replenishment of microbubbles compensates for the decay of signals from microbubbles 
1 due to disruption. Thus, signals from microbubbles can last longer until the end of data

2 acquisition. This explains both the qualitative results in Fig. 7 and results of CTR under flow velocities from $10 \mathrm{~mm} / \mathrm{s}$ to $80 \mathrm{~mm} / \mathrm{s}$.

Based on these qualitative and quantitative evaluation, it can be concluded that the image contrast is dependent on the combined effects of ultrasound pressure and flow velocity. In addition, the ability to retain contrast signals over time (i.e. persistence of contrast) is another important measure for the imaging performance. For low flow velocity, differentiation between microbubbles and tissue is difficult due to the similar spatiotemporal coherences. Under this condition, microbubble disruption introduced by increasing ultrasound pressure can significantly improve bubble contrast. But a compromise between bubble contrast and persistence should be achieved by optimising the MI value. At low MI, relatively stable microbubbles give insignificant enhancement in contrast quality. However, at high MI, bubble signal changes provide much higher contrast signals at the beginning of the acquisition but are decreasing over time due to the diminishing bubble disruption events as bubbles are destroyed with high ultrasound amplitude and replenishment of microbubbles is inadequate. For high flow velocity, contrast quality has already improved by the enhanced difference in spatial and temporal characteristics between fast flowing microbubbles and static tissue.

$\underline{\text { In vitro Ultrasound experiment: effect of probe motion }}$

Probe motion is common during practical ultrasound scan in clinic and it is necessary to localise SLN when tracking microbubbles flowing in lymphatic vessels. Hence, it is also important to consider probe motion in our study. Compared to experiments using a static probe, there is significant reduction of CTR for the moving probe for all the flow velocities as shown in Fig. 9 and Fig. 10a. This is because moving probe/tissue may share comparable spatiotemporal characteristics with flowing microbubbles. Hence, the suppression of clutter signal by STA is less efficient with probe motion especially for the slow flow velocities $(<10$ 
$1 \mathrm{~mm} / \mathrm{s}$ ). For this case, the efficacy of clutter filtering is improved with the help of motion

2 correction, where tissue motion resulting from moving probe is estimated and corrected first to

reduce the overlap in spatial and temporal domain between clutter and microbubble signals. This allows subsequent SVD filter to be more efficient in discriminating between echoes from tissue and microbubbles. The fast-flows on the other hand, even with probe/tissue motion, have adequate difference from tissue signals both in space and time for SVD filter to work. Accordingly, improvement is not found with the help of motion correction at higher flow rates.

It should be noted that the improvement of SVD filter in terms of filtering clutter signals under probe motion, in comparison with classic nonlinear sequence and high pass filters, has been shown in CEUS imaging(Desailly et al. 2017). In that study, much higher CTR was obtained at the same probe speed of $2 \mathrm{~mm} / \mathrm{s}$. That is likely due to the different application focus - in that study the diameter of the wall-less vessel in that tissue phantom was $5 \mathrm{~mm}$ which was much larger than the $300 \mu \mathrm{m}$ vessel in this lymphatic mimicking phantom. The contrast signal from flowing microbubbles in a much smaller vessel is certainly lower, which likely restricted the detection of vessel in the lymphatic phantom under probe motion.

\section{$\underline{\text { Optical experiments }}$}

Various phenomena of microbubbles insonified with low PRF focus ultrasound emission have been classified and reported with optical observation using high speed camera (de Jong et al. 2009; Postema et al. 2002; Postema et al. 2004b; Postema et al. 2004a; Zheng et al. 2007). In this paper, we employ similar optical method to study the signal persistence of microbubbles under slow flow conditions when exposed to ultrafast ultrasound plane wave imaging with a pulse repetition frequency of $5000 \mathrm{~Hz}$. With the help of optical observation, the trade-off between contrast and persistence due to different ultrasound pressures are better understood. Fast and violent coalescence, fragmentation, shrinking of microbubbles are induced by ultrasound with higher MI value while slow coalescence and bouncing of microbubbles are 
1 observed under moderate MI. Additionally, relatively stable microbubbles are detected under

2 low MI. Evaluation of persistence of contrast signals shows consistency between optical and

3 ultrasound results. One limitation with the optical experiment is that the performance of

4 microbubble was monitored in a capillary tube, which may cause changes in microbubble

5 characteristics and behaviour due to the wall proximity and complex acoustic environment in

6 the tube (Garbin et al. 2007).

$7 \quad$ Limitation and future works

In this study, all of the 1000 frames acquired in 1 second were used for STA processing, leading to long computation time. In an effort to implement real-time processing, shorter stacks of frames should be used for SVD filter. Hence, we further studied the filtering performance affected by different ensemble lengths (stack of frames). Both qualitative and quantitative results in Fig. 13 illustrated the profit gained from long ensemble length. Fig. 13a and Fig. 13b presented filtering results obtained by 1000 frames and 50 frames. Under the same flow velocity of $4 \mathrm{~mm} / \mathrm{s}$, larger stack of 1000 frames provided enhanced contrast. Fig. 13c illustrated CTR values achieved with various ensemble lengths and at different flow velocities. CTR decreased with the reduction of processing frame number. But at the highest flow velocity of $80 \mathrm{~mm} / \mathrm{s}$, all ensemble lengths share similar CTR values due to the unchallenging distinction between microbubble signals and tissue artefacts. Hence, the trade-off between image quality and processing time could be made according to the relative speed of flow and motion. Yann et al.(Desailly et al. 2017) proposed the idea of shortening SVD filter processing time by applying filtering on shorter ensemble length and showed qualitative result of a human kidney image by summing 30 frames $(30 \mathrm{~ms})$ with SVD filter processing. The advantage of large ensemble of frames in SVD filter has been further demonstrated quantitatively in in-vitro and in-vivo studies(Baranger et al. 2018; Song et al. 2017). 

included in this study and are worth investigating in future work. For instance, the frame rate gives a trade-off between the ability of capturing the slow lymph fluid and increased

microbubble destruction. Furthermore, SVD filter can be further optimised in terms of processing region and singular value threshold determination for in-vivo human imaging. Instead of global SVD, block-wise filtering technique can be used for the complicated spatial distribution of tissue and noise(Song et al. 2017). Adaptive methods for optimal thresholding can also be used to compare the efficiency in clutter and noise suppression(Baranger et al. 2018; Song et al. 2017).

Due to the practical difficulties associated with injecting the microbubbles into lymphatic system of rabbit, preliminary data of blood vasculature in rabbit popliteal lymph node were presented as an in vivo demonstration of slow flow imaging. Moderate MI (0.11) was chosen based on the in vitro study in order to achieve high contrast and better persistent signal in tracking the slow flow. Different from the in-intro experiments, higher imaging frequency was used in the rabbit experiments for the resolution requirement to image $\mathrm{LN}$ with diameter around 4-5 $\mathrm{mm}$. No motion correction was required as the rabbit is under anaesthesia and the hind limb as well as the probe were fixed.

\section{Conclusions}

This work applies HFR CEUS imaging to a lymph mimicking vessel and investigates the impacts of flow velocity, probe motion and ultrasound pressure on HFR CEUS for imaging flow in lymphatic vessel. Preliminary studies were conducted on imaging a rabbit popliteal lymph node. Our results provide evidence that significant tissue artefact in lymphatic imaging can be reduced by HFR and SVD filter at this high frequency range. We have shown that the optimal choice of MI value is related to the flow velocity. In particular, under high flow velocity image quality is less affected by MI value while MI plays a more significant role at low flow 
1 velocity. An applicable MI value can be chosen to reach a compromise between image contrast

2 and persistence/microbubble disruption under slow flow condition. Optical observation of 3 microbubble behaviour insonified with ultrafast plane wave imaging sequences has also been

4 studied to understand the importance of optimising signal persistence for imaging slow-flowing 5 lymphatic vessels. In the case of moving probe, significantly decreased contrast under lower 6 flow velocities can be improved by applying motion correction to the images prior to SVD filter.

7 HFR CEUS and SVD filter enabled visualisation of blood vessels inside rabbit popliteal lymph 8 node in which the blood flow velocities are less than 0 to $3 \mathrm{~mm} / \mathrm{s}$. 


\section{Acknowledgments}

This study was partially funded by the UK EPSRC under Grant Nos. EP/N015487/1, EP/N014855/1, and EP/M011933/1, the CRUK Multidisciplinary Project Award under Grant No. C53470/A22353, the Imperial Confidence-in-Concepts, the Imperial Proof-of-Concept and the BHF Centre of Research Excellence for funding. The authors thank Prof. Adrian Lim, Prof James E. Moore, Dr Robert J. Eckersley and Dr. Matthieu Toulemonde for their advice.

\section{References}

Baranger J, Arnal B, Perren F, Baud O, Tanter M, Demené C. Adaptive spatiotemporal SVD clutter filtering for Ultrafast Doppler Imaging using similarity of spatial singular vectors. IEEE Trans Med Imaging 2018;PP:1-1.

Bentel GC, Marks LB, Hardenbergh PH, Prosnitz LR. Variability of the depth of supraclavicular and axillary lymph nodes in patients with breast cancer: is a posterior axillary boost field necessary? Int J Radiat Oncol Biol Phys 2000;47:755-758.

Brock-Fisher GA, Poland MD, Rafter PG. Means for increasing sensitivity in non-linear ultrasound imaging systems. 1996 [cited 2018 Nov 26]. Available from: https://patents.google.com/patent/US5577505A/en

Couture O, Fink M, Tanter M. Ultrasound contrast plane wave imaging. IEEE Trans Ultrason Ferroelectr Freq Control 2012;59.

Cox K, Sever A, Jones S, Weeks J, Mills P, Devalia H, Fish D, Jones P. Validation of a technique using microbubbles and contrast enhanced ultrasound (CEUS) to biopsy sentinel lymph nodes (SLN) in pre-operative breast cancer patients with a normal grey-scale axillary ultrasound. Eur J Surg Oncol EJSO 2013;39:760-765.

de Jong N, Emmer M, van Wamel A, Versluis M. Ultrasonic characterization of ultrasound contrast agents. Med Biol Eng Comput 2009;47:861-873.

Demené C, Deffieux T, Pernot M, Osmanski BF, Biran V, Gennisson JL, Sieu LA, Bergel A, Franqui S, Correas JM, Cohen I, Baud O, Tanter M. Spatiotemporal Clutter Filtering of Ultrafast Ultrasound Data Highly Increases Doppler and fUltrasound Sensitivity. IEEE Trans Med Imaging 2015;34:2271-2285.

Demené C, Tiran E, Sieu L-A, Bergel A, Gennisson JL, Pernot M, Deffieux T, Cohen I, Tanter M. 4D microvascular imaging based on ultrafast Doppler tomography. NeuroImage 2016;127:472-483.

Denarie B, Tangen TA, Ekroll IK, Rolim N, Torp H, Bjåstad T, Lovstakken L. Coherent Plane Wave Compounding for Very High Frame Rate Ultrasonography of Rapidly Moving Targets. IEEE Trans Med Imaging 2013;32:1265-1276. 
Desailly Y, Tissier A-M, Correas J-M, Wintzenrieth F, Tanter M, Olivier Couture. Contrast enhanced ultrasound by real-time spatiotemporal filtering of ultrafast images. Phys Med Biol 2017;62:31.

Fujishima M, Watatani M, Inui H, Hashimoto Y, Yamamoto N, Hojo T, Hirai K, Yamato M, Shiozaki $\mathrm{H}$. Touch imprint cytology with cytokeratin immunostaining versus Papanicolau staining for intraoperative evaluation of sentinel lymph node metastasis in clinically node-negative breast cancer. Eur J Surg Oncol EJSO 2009;35:398-402.

Garbin V, Cojoc D, Ferrari E, Di Fabrizio E, Overvelde MLJ, van der Meer SM, de Jong N, Lohse D, Versluis M. Changes in microbubble dynamics near a boundary revealed by combined optical micromanipulation and high-speed imaging. Appl Phys Lett 2007;90:114103.

Goldberg BB, Merton DA, Liu J-B, Thakur M, Murphy GF, Needleman L, Tornes A, Forsberg F. Sentinel Lymph Nodes in a Swine Model with Melanoma: Contrastenhanced Lymphatic US. Radiology 2004;230:727-734.

Harput S, Christensen-Jeffries K, Li Y, Brown J, Eckersley RJ, Dunsby C, Tang MX. Two stage sub-wavelength motion correction in human microvasculature for CEUS imaging. 2017 IEEE Int Ultrason Symp IUS 2017. pp. 1-4.

Lin S, Zhang G, Leow CH, Tang M-X. Effects of microchannel confinement on acoustic vaporisation of ultrasound phase change contrast agents. Phys Med Biol 2017;62:6884.

Madsen EL, Frank GR, Dong F. Liquid or Solid Ultrasonically Tissue-Mimicking Materials with Very Low Scatter. Ultrasound Med Biol 1998;24:535-542.

Montaldo G, Tanter M, Bercoff J, Benech N, Fink M. Coherent plane-wave compounding for very high frame rate ultrasonography and transient elastography. IEEE Trans Ultrason Ferroelectr Freq Control 2009;56:489-506.

Pan W-R, le Roux CM, Levy SM, Briggs CA. The morphology of the human lymphatic vessels in the head and neck. Clin Anat 2010;23:654-661.

Pesek S, Ashikaga T, Krag LE, Krag D. The false-negative rate of sentinel node biopsy in patients with breast cancer: a meta-analysis. World J Surg 2012;36:2239-2251.

Postema M, Bouakaz A, Chin CT, Jong N de. Optically observed microbubble coalescence and collapse. 2002 IEEE Ultrason Symp 2002 Proc 2002. pp. 1949-1952 vol.2.

Postema M, Marmottant P, Lancée CT, Hilgenfeldt S, Jong N de. Ultrasound-induced microbubble coalescence. Ultrasound Med Biol 2004a;30:1337-1344.

Postema M, van Wamel A, Lancée CT, de Jong N. Ultrasound-induced encapsulated microbubble phenomena. Ultrasound Med Biol 2004b;30:827-840.

Sever AR, Mills P, Jones SE, Cox K, Weeks J, Fish D, Jones PA. Preoperative sentinel node identification with ultrasound using microbubbles in patients with breast cancer. AJR Am J Roentgenol 2011;196:251-256. 
Simpson DH, Chin CT, Burns PN. Pulse inversion Doppler: a new method for detecting nonlinear echoes from microbubble contrast agents. IEEE Trans Ultrason Ferroelectr Freq Control 1999;46:372-382.

Somasundaram SK, Chicken DW, Keshtgar MRS. Detection of the sentinel lymph node in breast cancer. Br Med Bull 2007;84:117-131.

Song P, Manduca A, Trzasko JD, Chen S. Ultrasound Small Vessel Imaging With BlockWise Adaptive Local Clutter Filtering. IEEE Trans Med Imaging 2017;36:251-262.

Tang M-X, Kamiyama N, Eckersley RJ. Effects of Nonlinear Propagation in Ultrasound Contrast Agent Imaging. Ultrasound Med Biol 2010;36:459-466.

Tanter M, Fink M. Ultrafast imaging in biomedical ultrasound. IEEE Trans Ultrason Ferroelectr Freq Control 2014;61:102-119.

Temple LKF, Baron R, Cody HS, Fey JV, Thaler HT, Borgen PI, Heerdt AS, Montgomery LL, Petrek JA, Van Zee KJ. Sensory morbidity after sentinel lymph node biopsy and axillary dissection: a prospective study of 233 women. Ann Surg Oncol 2002;9:654662.

van der Ven M, Luime JJ, van der Velden LL, Bosch JG, Hazes JMW, Vos HJ. High-FrameRate Power Doppler Ultrasound Is More Sensitive than Conventional Power Doppler in Detecting Rheumatic Vascularisation. Ultrasound Med Biol 2017;43:1868-1879.

Veronesi U, Paganelli G, Viale G, Luini A, Luini S, Galimberti V, Intra M, Veronesi P, Robertson C, Maisonneuve P, Renne G, De Cicco C, De Lucia F, Gennari R. A Randomized Comparison of Sentinel-Node Biopsy with Routine Axillary Dissection in Breast Cancer. N Engl J Med 2003;349:546-553.

Wong J, Yong WS, Thike AA, Iqbal J, Salahuddin AS, Ho GH, Madhukumar P, Tan BKT, Ong KW, Tan PH. False negative rate for intraoperative sentinel lymph node frozen section in patients with breast cancer: a retrospective analysis of patients in a single Asian institution. J Clin Pathol 2015;68:536-540.

Zheng H, Dayton PA, Caskey C, Zhao S, Qin S, Ferrara KW. Ultrasound-Driven Microbubble Oscillation and Translation Within Small Phantom Vessels. Ultrasound Med Biol 2007;33:1978-1987.

Nanomedicine, Volume I: Basic Capabilities. CRC Press. 1999 [cited 2017 Aug 23]. Available from: https://www.crcpress.com/Nanomedicine-Volume-I-BasicCapabilities/Freitas/p/book/9781570596803 


\section{Figure Captions List}

Figure 1. (a) A CEUS image of human axilla at 7MHz containing nonlinear tissue artefacts (AF, arrows), making identification of the microbubble-filled lymph vessel (LV, arrow) difficult; (b) A CEUS image of a human lymph node (LN, arrow). Scales on the left of the images are in $\mathrm{cm}$. Figure 2. (a) In-vivo set-up (not to scale) for investigating the effects of flow velocity and ultrasound pressure. Tissue-mimicking phantom with a $300 \mu \mathrm{m}$ tube was designed to simulate the size and depth of lymphatic vessel. Microbubbles were injected by a syringe pump to achieve velocities in the range of lymph flow. Ultrasound probe was mounted on a linear motor to simulate probe motion. (b) In-vitro two-tube set-up (not to scale) for comparing PI and SVD filter, the bottom $200 \mu \mathrm{m}$ cellulose tube was filled with non-flowing microbubbles and the top tube was filled with water as control.

Figure 3. In-vitro set-up (not to scale) for optically observing microbubble behaviours under varied MI: Optical microscope connected with high-speed camera was used to monitor microbubble disruption with a frame rate of 12000 fps. The high-speed camera was synchronized with ultrasound image sequences. The optical path and ultrasound field were set up normal to each other to eliminate the effect on ultrasound wave from the object in the optical path.

Figure 4. Qualitative comparison between PI and SVD filtered ultrasound images. SVD filtered image was able to detect bubbles from the bottom tube while removing nonlinear signals from the control tube.

Figure 5. Qualitative evaluation of the effects of flow velocities in static probe cases: ultrasound images after STA on B-mode images at a fixed MI of 0.05 and under increasing flow velocities (a) $4 \mathrm{~mm} / \mathrm{s}$, (b) $10 \mathrm{~mm} / \mathrm{s}$, (c) $20 \mathrm{~mm} / \mathrm{s}$, (d) $40 \mathrm{~mm} / \mathrm{s}$, (e) $80 \mathrm{~mm} / \mathrm{s}$. 
Figure 6. Qualitative evaluation of the effects of ultrasound pressure: ultrasound images after SVD filter on B-mode images under fixed flow velocity of $4 \mathrm{~mm} / \mathrm{s}$ and at increased MIs and two time points (a) $\mathrm{MI}=0.05$ Time $=50 \mathrm{~ms}$, (b) $\mathrm{MI}=0.05$ Time $=550 \mathrm{~ms}$, (c) $\mathrm{MI}=0.11$ Time=50 ms, (d) MI=0.11 Time=550 ms, (e) MI=0.22 Time=50 ms, (f) MI=0.22 Time=550 ms.

Figure 7. Qualitative evaluation of the effects of ultrasound pressure: ultrasound images after SVD filter on B-mode images under fixed flow velocity of $10 \mathrm{~mm} / \mathrm{s}$ and at increased MIs and two time points (a) $\mathrm{MI}=0.05$ Time $=50 \mathrm{~ms}$, (b) $\mathrm{MI}=0.05$ Time $=550 \mathrm{~ms}$, (c) $\mathrm{MI}=0.11$ Time $=50$ $\mathrm{ms}$, (d) MI=0.11 Time=550 ms, (e) MI=0.22 Time=50 ms, (f) MI=0.22 Time $=550 \mathrm{~ms}$.

Figure 8. (a) Quantitative evaluation of effects of flow velocity and ultrasound pressure by CTR. (b) Quantitative evaluation of the effects of ultrasound pressure on INI.

Figure 9. Qualitative evaluation of effects of probe motion: ultrasound images after SVD filter on B-mode images at a fixed MI of 0.11 and under increased flow velocities and probe speed (a) flow velocity $=4 \mathrm{~mm} / \mathrm{s}$, probe speed $=0$ (b) flow velocity $=4 \mathrm{~mm} / \mathrm{s}$, probe speed $=2 \mathrm{~mm} / \mathrm{s}$ (c) flow velocity $=10 \mathrm{~mm} / \mathrm{s}$, probe speed $=0$ (d) flow velocity $=10 \mathrm{~mm} / \mathrm{s}$, probe speed $=2$ $\mathrm{mm} / \mathrm{s}$ (e) flow velocity $=20 \mathrm{~mm} / \mathrm{s}$, probe speed $=0$ (f) flow velocity $=20 \mathrm{~mm} / \mathrm{s}$, probe speed $=2 \mathrm{~mm} / \mathrm{s}$.

Figure 10. (a) Quantitative comparison between static probe and moving probe by CTR. (b) Effect of motion correction on CTR at different velocities.

Figure 11. Optical observation of microbubble behaviours (a) at MI of 0.36, violent and rapid changes of microbubbles including multiple coalescence, shrinking and translation of microbubbles. (b) at MI of 0.36 including repetitive fragmentation and coalescence, shrinking and translation of microbubbles. (c) at MI of 0.22 , the main observed behaviour is coalescence. (d) at MI of 0.11 , most microbubbles just attached to each other without further combining process. (e) at MI of 0.11, a few microbubbles coalesced. 
Figure 12. HFR CEUS images of rabbit popliteal lymph node (a) B-mode image (b) Vascular image (c) Directional image. Blue colour indicates flow going towards the transducer and red colour indicates flow going away from transducer. The colourmap reveals the velocities in $\mathrm{mm} / \mathrm{s}$.

Figure 13. Qualitative evaluation of effect of frame number on SVD filtered images. (a) STA applied on 1000 frames, (b) SVD filter applied on 50 frames. (c) Quantitative evaluation of effect of frame number on SVD filter by CTR.

Table. 1. Comparison of microbubble disruption between different ultrasound pressures. Disruption of microbubbles is defined as changes of microbubbles including coalescence, fragmentation, shrink and destruction.

\begin{tabular}{|l|l|l|}
\hline \multirow{2}{*}{ Ultrasound pressure } & \multicolumn{2}{|l|}{$\begin{array}{l}\text { Number of disrupted microbubbles / Total number of } \\
\text { microbubbles }\end{array}$} \\
\cline { 2 - 3 } & $50 \mathrm{~ms}$ (in \%) & $550 \mathrm{~ms}$ (in \%) \\
\hline $\begin{array}{l}\mathrm{MI}=0.36(\mathrm{PNP}=0.64 \mathrm{MPa} \text { at } \\
\text { 4MHz) }\end{array}$ & $100.00 \pm 0.00$ & $100.00 \pm 0.00$ \\
\hline $\mathrm{MI}=0.2(\mathrm{PNP}=0.40 \mathrm{MPa})$ & $49.50 \pm 11.43$ & $71.45 \pm 12.37$ \\
\hline $\mathrm{MI}=0.1(\mathrm{PNP}=0.20 \mathrm{MPa})$ & $0.00 \pm 0.00$ & $23.21 \pm 9.28$ \\
\hline
\end{tabular}


Figure 1:

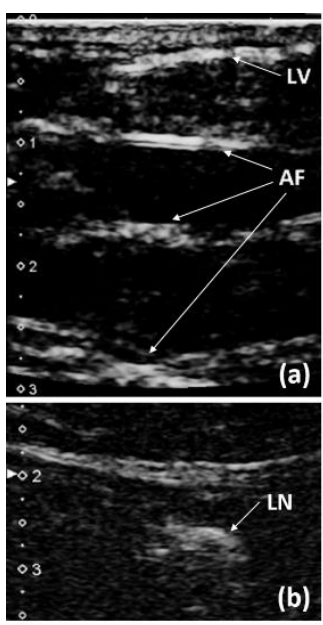

Figure 2:
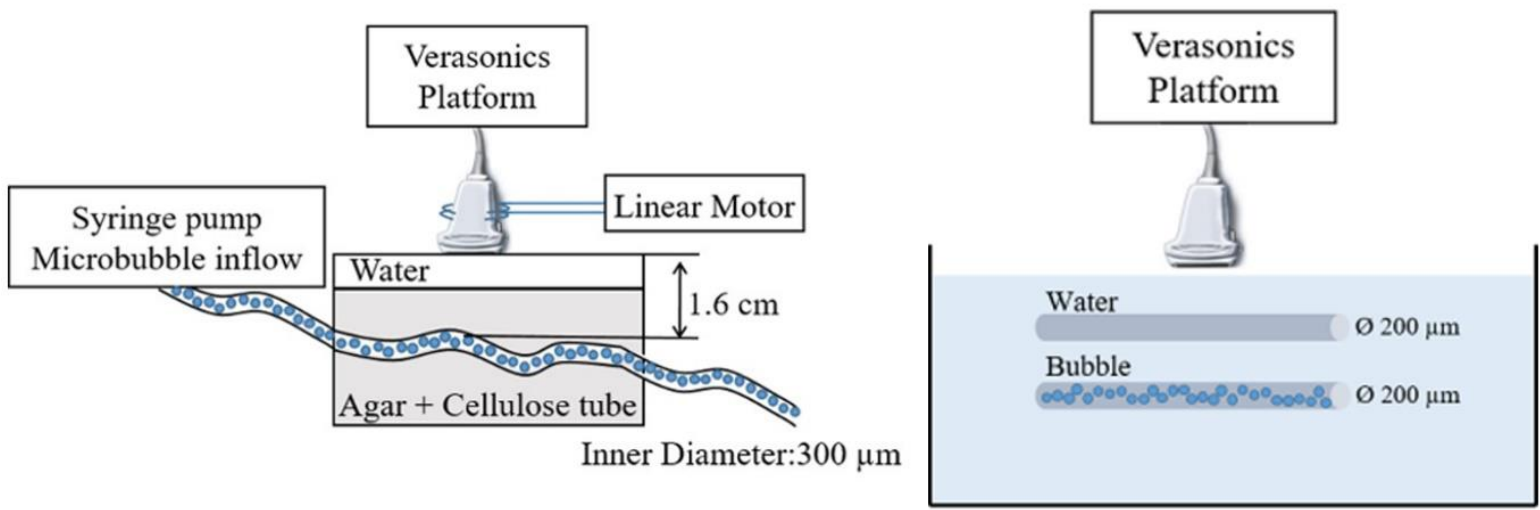

(a)

(b)

Figure 3

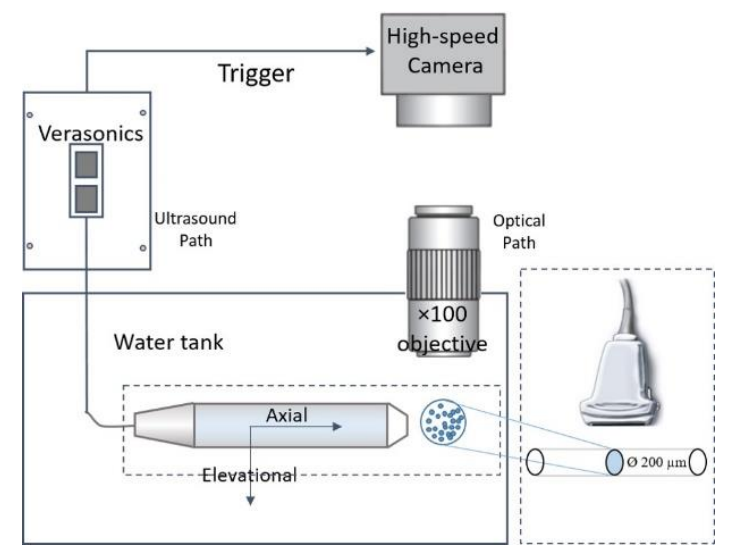


Figure 4

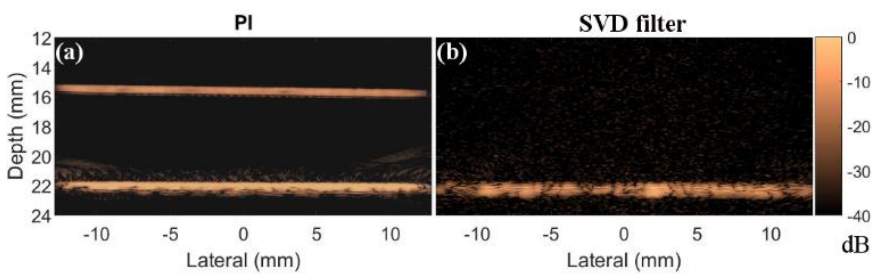

Figure 5

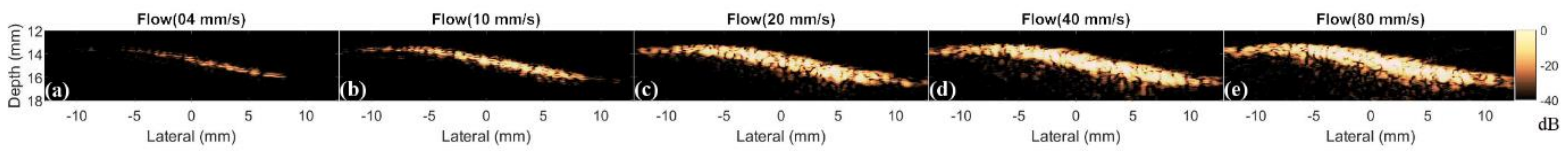

Figure 6

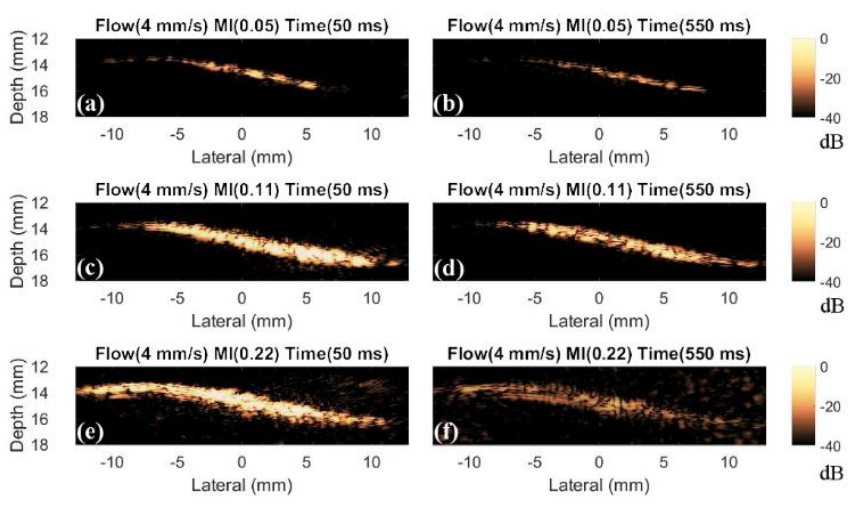

Figure 7
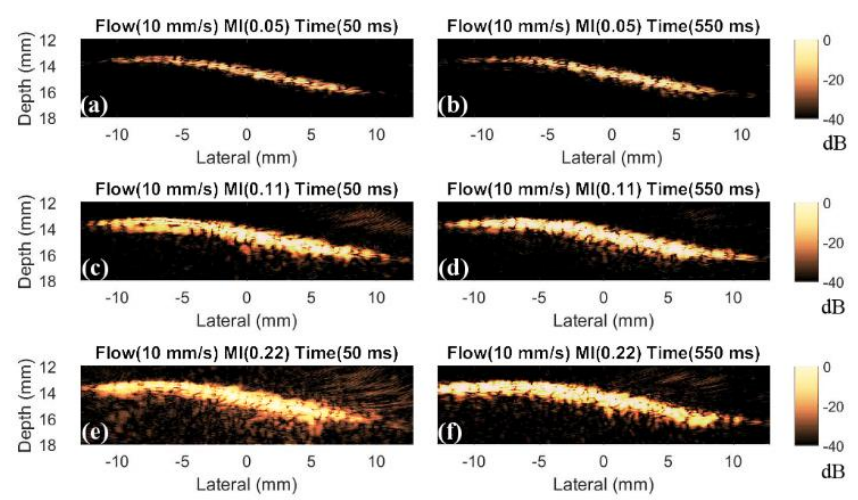

Figure 8 


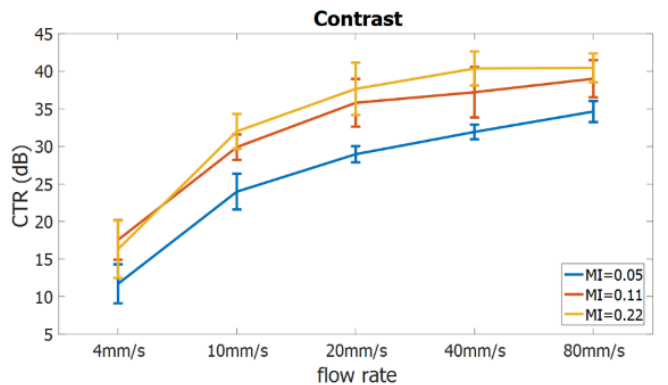

(a)

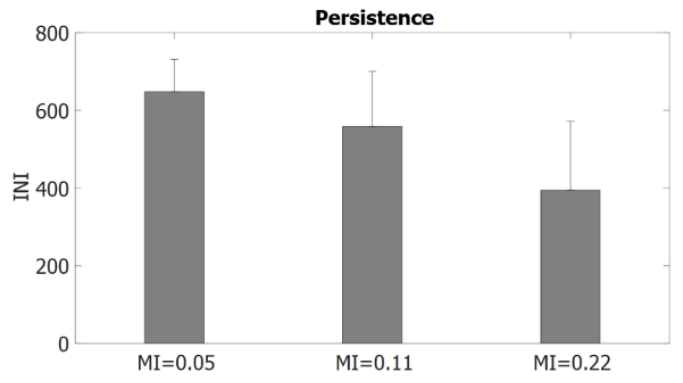

(b)

Figure 9

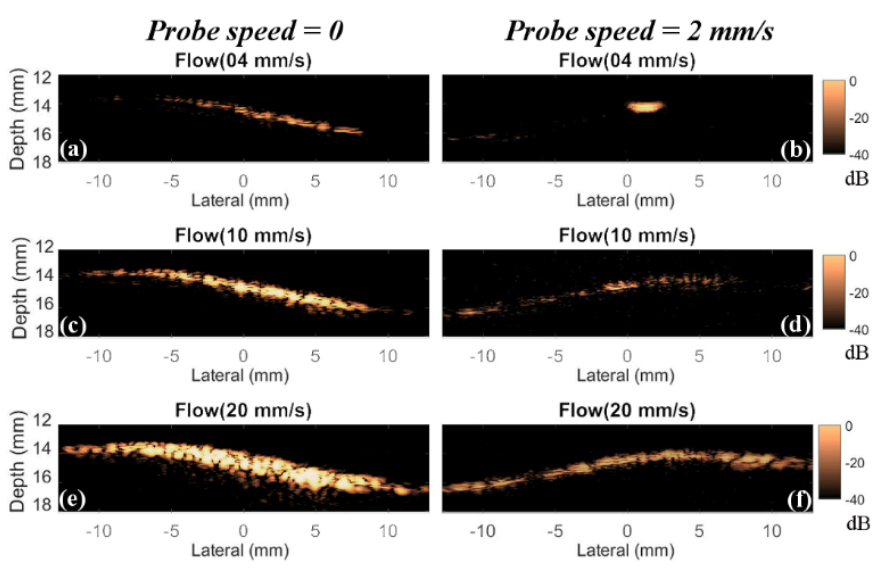

Figure 10

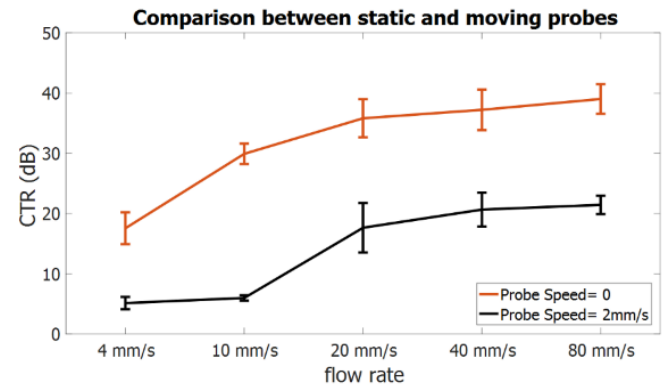

(a)

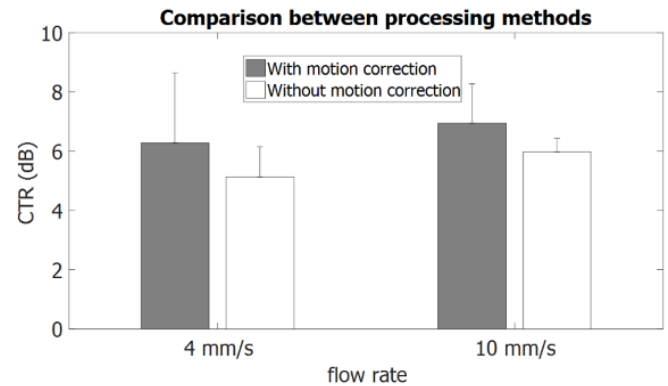

(b) 
Figure 11

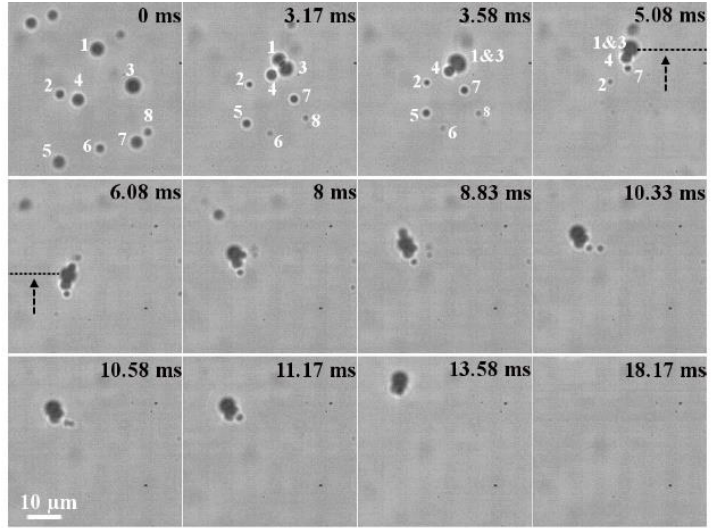

(a)

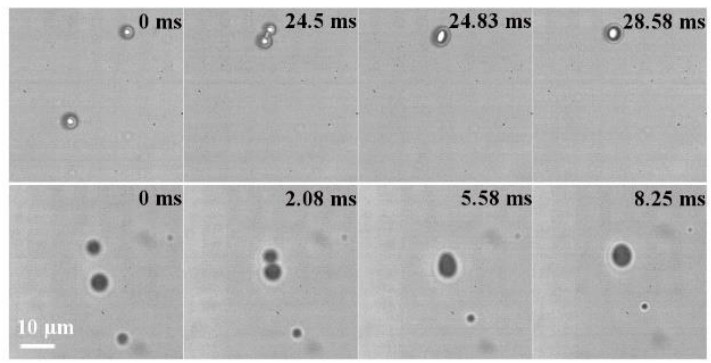

(c)

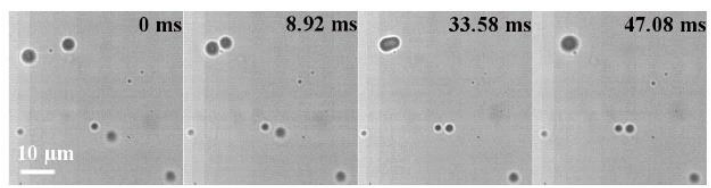

(e)

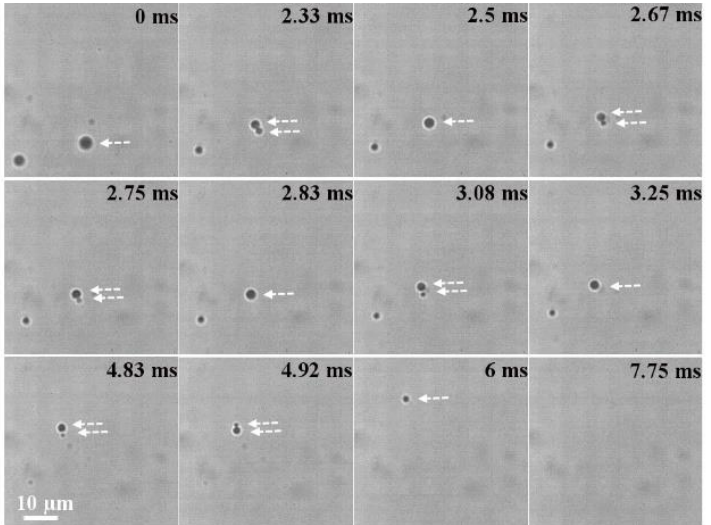

(b)

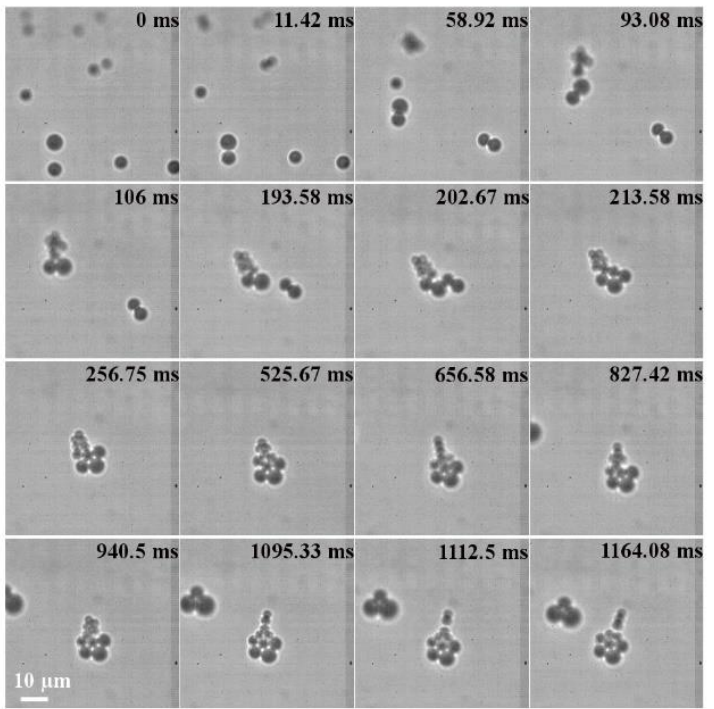

(d)

Figure 12

(a)

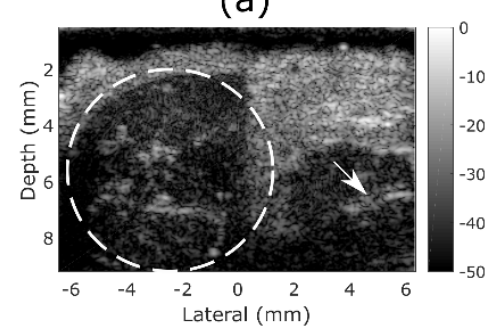

(b)

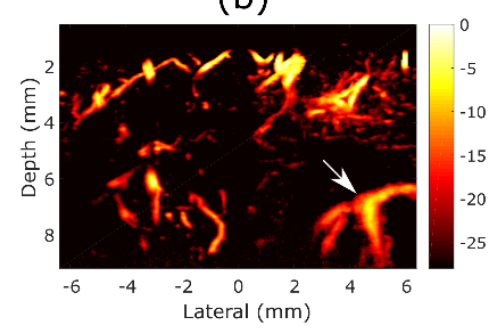

(c)

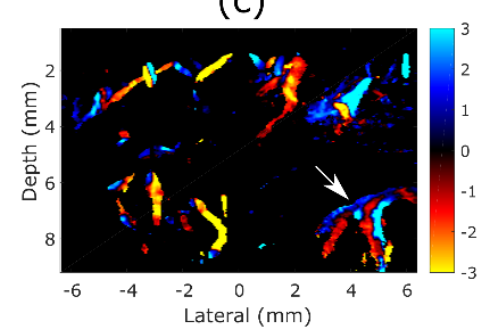


Figure 13

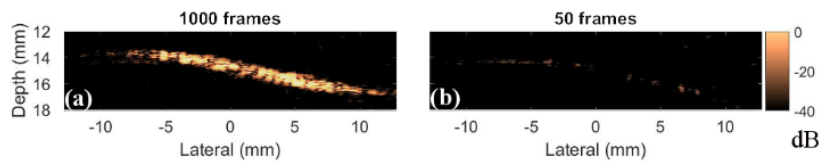

(c)

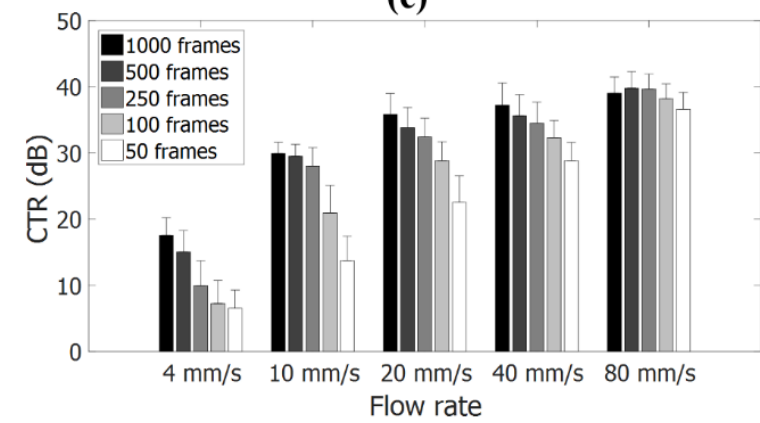

